\title{
Author Correction: Atomically precise graphene etch stops for three dimensional integrated systems from two dimensional material heterostructures
}

Jangyup Son ${ }^{1}$, Junyoung Kwon², SunPhil Kim¹, Yinchuan Lv³, Jaehyung Yư ${ }^{1}$, Jong-Young Lee ${ }^{2}$, Huije Ryu ${ }^{2}$ Kenji Watanabe (1) 4, Takashi Taniguchi ${ }^{4}$, Rita Garrido-Menacho ${ }^{3,5}$, Nadya Mason ${ }^{3,5}$, Elif Ertekin ${ }^{1,5}$, Pinshane Y. Huang 5,6 , Gwan-Hyoung Lee (10) ${ }^{2}$ \& Arend M. van der Zande ${ }^{1,5}$

Correction to: Nature Communications; https://doi.org/10.1038/s41467-018-06524-3; published online 28 September 2018

The original version of this Article contained an error in the second sentence of the second paragraph of the 'Electrical properties of fluorinated graphene contacts' section of the Results, which incorrectly read 'The mobility was calculated by the Drude model, $\mu=n e / \sigma$ where $\mu, n, e$, and $\sigma$ are the carrier mobility, carrier density, electron charge, and sheet conductivity, respectively'. The correct version states ' $\mu=\sigma / n e$ ' in place of ' $\mu=n e / \sigma$ '. This has been corrected in both the PDF and HTML versions of the Article.

Published online: 20 November 2018

(i) Open Access This article is licensed under a Creative Commons Attribution 4.0 International License, which permits use, sharing, adaptation, distribution and reproduction in any medium or format, as long as you give appropriate credit to the original author(s) and the source, provide a link to the Creative Commons license, and indicate if changes were made. The images or other third party material in this article are included in the article's Creative Commons license, unless indicated otherwise in a credit line to the material. If material is not included in the article's Creative Commons license and your intended use is not permitted by statutory regulation or exceeds the permitted use, you will need to obtain permission directly from the copyright holder. To view a copy of this license, visit http://creativecommons.org/licenses/by/4.0/.

(C) The Author(s) 2018

\footnotetext{
${ }^{1}$ Department of Mechanical Science and Engineering, University of Illinois at Urbana-Champaign, 1206 W Green Street, Urbana, IL 61801, USA. ${ }^{2}$ Department of Materials Science and Engineering, Yonsei University, 50 Yonsei-ro, Seodaemun-gu, Seoul 03722, Korea. ${ }^{3}$ Department of Physics, University of Illinois at Urbana-Champaign, 1110 W Green Street, Urbana, IL 61801, USA. ${ }^{4}$ National Institute for Materials Science, 1-1 Namiki, Tsukuba, Ibaraki 305-0044, Japan. ${ }^{5}$ Frederick Seitz Materials Research Laboratory, University of Illinois at Urbana-Champaign, 104 S Goodwin Avenue MC-230, Urbana, IL 61801, USA. ${ }^{6}$ Department of Materials Science and Engineering, University of Illinois at Urbana-Champaign, 1304 W Green Street, Urbana, IL 61801, USA. 